\title{
COMPOSIÇÃO QUÍMICA DO CAPIM-MOMBAÇA (Panicum maximum Jacq.) SUBMETIDO À ADUBAÇÃO ORGÂNICA E MINERAL
}

\author{
Karina Rocha Freit As ${ }^{1}$, BenEVAl Rosa ${ }^{2}$, JoRge Luiz do NASCIMENTO ${ }^{3}$, RODRIGO TAVARES \\ BORGES $^{4}$, MAisa Matias Barbosa ${ }^{5}$, DARliane De CASTRO SANTOS ${ }^{6}$ \\ 1 - Professora Doutora, Instituto Federal de Educação, Ciência e Tecnologia de Goiás - Inhumas, GO - freitaskk@yahoo.com.br \\ 2 - Professor Doutor, Escola de Veterinária e Zootecnia da Universidade Federal de Goiás - Goiânia, GO. \\ 3 - Professor Doutor, Escola de Agronomia da Universidade Federal de Goiás Goiás - Goiânia, GO. \\ 4 - Mestrando em Produção Vegetal da Escola de Agronomia da UFG Goiás - Goiânia, GO. \\ 5 - Acadêmica do curso de Medicina Veterinária (EVZ-UFG) - Goiânia, GO. \\ 6 - Acadêmica do curso de Agronomia (UFG) e bolsista (PIVIC/CNPq) Goiás - Goiânia, GO.
}

\section{RESUMO}

Realizou-se o trabalho com o objetivo de avaliar a composição química de Panicum maximum Jacq. cv. Mombaça fertilizado com dejetos líquidos de suínos (DLS) e adubo mineral na época das chuvas durante dois anos consecutivos. O experimento foi conduzido no período de outubro de 2004 a março de 2005 e no período de setembro de 2005 a abril de 2006. Utilizaram-se os seguintes tratamentos: Testemunha=reposição de 3,5 $\mathrm{kg} / \mathrm{ha}$ de $\mathrm{P}_{2} \mathrm{O}_{5}$ e $18 \mathrm{~kg} / \mathrm{ha}$ de $\mathrm{K}_{2} \mathrm{O} / \mathrm{t}$ de massa seca de forragem colhida; $\mathrm{TQ}=$ reposição de $3,5 \mathrm{~kg} / \mathrm{ha}$ de $\mathrm{P}_{2} \mathrm{O}_{5}, 18$ $\mathrm{kg} / \mathrm{ha}$ de $\mathrm{K}_{2} \mathrm{O} / \mathrm{t}$ de massa seca de forragem colhida e 300 kg de N/ha/ano; T100=100 m³/ha/ano de DLS; T150=150 $\mathrm{m}^{3} / \mathrm{ha} /$ ano de DLS; T200=200 $\mathrm{m}^{3} / \mathrm{ha} /$ ano de DLS. As fertilizações foram parceladas pelo número de cortes realizados em cada ano e aplicadas após cada corte. Os cortes foram realizados a cada 28 dias a $0,30 \mathrm{~m}$ do solo. Foram determinados os macrominerais (N, P, K, S, Ca, $\mathrm{Mg}$ ) e os microminerais ( $\mathrm{Cu}, \mathrm{Fe}, \mathrm{Zn}$ e $\mathrm{Mn}$ ) em cada período avaliado. O delineamento experimental utilizado foi o de blocos casualizados com parcelas subdivididas no tempo. As parcelas foram constituídas pelos tratamentos de fertilização e as sub-parcelas pelos anos de avaliação. A composição química foi influenciada tanto pela fertilização com DLS e mineral, quanto pelos anos de avaliação. A melhor resposta foi com a dose de 200 $\mathrm{m}^{3} / \mathrm{ha} / \mathrm{ano}$ de DLS.

PALAVRAS-CHAVE: fertilização orgânica; minerais; pastagem.

CHEMICAL COMPOSITION OF Panicum maximum Jacq. SUBMITTED TO ORGANIC AND MINERAL FERTILIZATION

\section{ABSTRACT}

The objective of this study was to evaluate the chemical composition of Panicum maximum Jacq. fertilized with pig slurry (PS) and mineral fertilizer during the rainy season for two consecutive years. The experiment was conducted from October 2004 to March 2005 and from September 2005 to April 2006. The following treatments were used: Witness=replacement of $3.5 \mathrm{~kg} / \mathrm{ha}$ of $\mathrm{P}_{2} \mathrm{O}_{5}$ and $18 \mathrm{~kg} / \mathrm{ha}$ of $\mathrm{K}_{2} \mathrm{O} / \mathrm{t}$ of dry matter of forage;
TQ=replacement of $3.5 \mathrm{~kg} / \mathrm{ha}$ of $\mathrm{P}_{2} \mathrm{O}_{5}, 18 \mathrm{~kg} / \mathrm{ha}$ of $\mathrm{K}_{2} \mathrm{O} / \mathrm{t}$ of dry matter of forage and $300 \mathrm{~kg}$ of $\mathrm{N} / \mathrm{ha} / \mathrm{year}$; $\mathrm{T} 100=100 \mathrm{~m}^{3} / \mathrm{ha} /$ year of LPW; T150=150 $\mathrm{m}^{3} /$ ha/year of LPW; T200=200 $\mathrm{m}^{3} / \mathrm{ha} /$ year of LPW. The fertilizations were parceled out by the number of cuttings accomplished every year and applied after each cut. The cuttings were accomplished every 28 days at $0.30 \mathrm{~m}$ of the soil. Macromineral (N, P, K, S, Ca, Mg) and microminerals 
(Cu, Fe, Zn e Mn) were assessed in each evaluated period. The experimental design was split-plot random blocks, constituted by the fertilization treatments and by the years of evaluation. The chemical composition was influenced

KEYWORDS: grass; minerals; organic fertilization.

\section{INTRODUÇÃO}

No Brasil, a intensificação da fertilização com adubo mineral passou a ser uma das maiores inovações tecnológicas nas décadas de 50 a 70 e, como consequência, o uso da fertilização orgânica nesse período foi quase que totalmente esquecido (MARRIEL et al., 1987). A agricultura iniciou, então, uma fase de elevada dependência de insumos, que perdura até os dias de hoje (KIEHL, 1997).

Alguns fatores, como o custo elevado de fertilizantes químicos, a sua disponibilidade limitada em regiões distantes dos centros de produção e a redução da capacidade produtiva dos solos, em razão do uso inadequado de fertilizantes químicos, representam um desafio à produção de alimentos em qualidade e quantidade suficientes para atender a crescente demanda desses produtos (MARRIEL et al., 1987). Atualmente, é reconhecida a necessidade de conservação dos recursos naturais e de preservação do meio ambiente, assim como do despertar em toda a sociedade de uma conscientização ecológica.

Dentro dessa visão, um dos principais problemas na atividade da suinocultura origina-se das dificuldades de manejo e destino dos dejetos produzidos pela crescente concentração geográfica da produção animal e da intensificação através de sistemas de confinamento.

Em 2006, Goiás detinha cerca de 58 mil matrizes suínas em produção, gerando em torno de 3,2 milhões de $\mathrm{m}^{3}$ de dejetos líquidos/ano (Konzen, 2006), sendo que estes têm alto poder poluente, especialmente para os recursos hídricos, em termos de demanda bioquímica de oxigênio (DBO). A alternativa mais utilizada para o aproveitamento dos dejetos líquidos de suínos (DLS) é na fertilização de pastagens e de culturas.

Os DLS são excelentes fontes de nutrientes, principalmente nitrogênio, fósforo e potássio (ADELI \& VARCO, 2001; ECHBERG, 2003; MENEZES et al., 2003) e, quando manejados corretamente, podem substituir parcial ou totalmente by the fertilization with LPW and mineral, as well as by the years of evaluation. The use of $200 \mathrm{~m}^{3} /$ ha/ano dose of DLS is recommended.

o fertilizante químico.

Rosa et al. (2004a) avaliaram o efeito da utilização de DLS (100, 150 e $\left.200 \mathrm{~m}^{3} / \mathrm{ha} / \mathrm{ano}\right)$ na recuperação de pastagens de capim-Marandu e não observaram influência da fertilização orgânica com DLS ou adubo mineral na composição química da forrageira, com exceção do elemento potássio (K), que apresentou teores mais elevados com a aplicação de 150 e $200 \mathrm{~m}^{3} / \mathrm{ha} / \mathrm{ano}$ de DLS. Em outro estudo, avaliando os mesmos tratamentos, ROSA et al. (2004 b), constataram que a fertilização com DLS e adubo mineral não influenciaram a composição química do capim-Marandu, somente os teores de manganês $(\mathrm{Mn})$ elevaram-se com a utilização do adubo mineral.

O capim-Mombaça (Panicum maximum Jacq.) é considerado uma das forrageiras tropicais mais produtivas, podendo atingir PMS anual em torno de 33 t/ha (JANK et al., 1994) e ainda apresentar satisfatória composição química. Atualmente, não há informações científicas sobre a utilização de DLS em pastagens de capim-Mombaça, porém muitos produtores utilizam-se dessa prática. Torna-se necessário saber quais os efeitos da fertilização com DLS quando comparada com o adubo mineral em relação à composição química da forrageira.

Realizou-se o presente estudo com o objetivo de avaliar a composição química de Panicum maximum Jacq. cv. Mombaça fertilizado com dejetos líquidos de suínos e adubo mineral na época das chuvas durante dois anos consecutivos.

\section{MATERIAL E MÉTODOS}

O experimento foi conduzido nas dependências da Escola de Agronomia da Universidade Federal de Goiás (EA/UFG), localizada no município de Goiânia. A área de pastagem de capim-Mombaça foi implantada em janeiro de 2002 em Latossolo Vermelho Distrófico Argissólico de textura média. Antes do início do experimento (Março/2004), fez-se análise de solo para verificação das suas características químicas, 
cujo resultado está expresso na Tabela 1.

TABELA 1 - Características químicas do solo com capim-Mombaça, na profundidade de 0-0,10 m

\begin{tabular}{|c|c|c|c|c|c|c|c|}
\hline $\mathrm{pH}$ & $\mathrm{Ca}$ & $\begin{array}{ll}\mathrm{Mg} & \mathrm{Al}\end{array}$ & $\mathrm{H}+\mathrm{Al}$ & CTC & $\mathrm{P}^{*}$ & MO & V \\
\hline $\mathrm{CaCl}_{2}$ & & $\mathrm{cmol}$ & $/ \mathrm{dm}^{3}$ & & $\mathrm{mg} / \mathrm{dm}^{3}$ & $\mathrm{~g} / \mathrm{kg}$ & $\%$ \\
\hline 5,2 & 2,9 & $1,1 \quad 0,0$ & 5,3 & 9,6 & 2,6 & 11 & 47,4 \\
\hline
\end{tabular}

Considerando os resultados apresentados anteriormente, fez-se a correção da fertilidade do solo em julho de 2004, com aplicação a lanço de 0,45 t/ha de calcário Filer (PRNT $=130 \%$ ), a fim de elevar a saturação por bases para $60 \%, 105 \mathrm{~kg} / \mathrm{ha}$ de $\mathrm{P}_{2} \mathrm{O}_{5}$ (superfosfato simples) e $50 \mathrm{~kg} / \mathrm{ha}$ de FTE BR 12 , seguindo as recomendações de VILELA et al. (2004).

O período experimental compreendeu a época das chuvas de dois anos consecutivos, sendo de outubro/2004 a março/2005 e de setembro/2005 a abril/2006. Os cortes foram realizados nas parcelas a cada 28 dias durante a época das águas por dois anos, sendo que a forragem foi cortada com cutelo a uma altura de $30 \mathrm{~cm}$ do solo, seguindo metodologia proposta por FAVORETTO et al. (1994). A área útil avaliada constituiu-se das cinco linhas internas (com 1 metro de comprimento cada), perfazendo uma área de $1,5 \mathrm{~m}^{2}$. Após cada corte de avaliação da forrageira, foi realizado o corte das bordaduras da área experimental $(0,30 \mathrm{~m}$ do solo) com roçadeira costal e, em seguida, os resíduos eram rastelados e retirados de toda a área experimental.

Utilizaram-se os seguintes tratamentos de fertilização:

- Testemunha=reposição de $3,5 \mathrm{~kg} /$ ha de $\mathrm{P}_{2} \mathrm{O}_{5}$ e $18 \mathrm{~kg} / \mathrm{ha}$ de $\mathrm{K}_{2} \mathrm{O} / \mathrm{t}$ de massa seca de forragem colhida;

- $\quad \mathrm{TQ}=$ reposição de $3,5 \mathrm{~kg} / \mathrm{ha}$ de $\mathrm{P}_{2} \mathrm{O}_{5}, 18$ $\mathrm{kg} / \mathrm{ha}$ de $\mathrm{K}_{2} \mathrm{O} / \mathrm{t}$ de massa seca de forragem colhida e $300 \mathrm{~kg} / \mathrm{ha} / \mathrm{ano}$ de $\mathrm{N}$;

- $\mathrm{T} 100=100 \mathrm{~m}^{3} / \mathrm{ha} / \mathrm{ano}$ de dejetos líquidos de suínos;

- $\mathrm{T} 150=150 \mathrm{~m}^{3} / \mathrm{ha} / \mathrm{ano}$ de dejetos líquidos de suínos;

- $\mathrm{T} 200=200 \mathrm{~m}^{3} / \mathrm{ha} / \mathrm{ano}$ de dejetos líquidos de suínos.

As aplicações dos DLS, bem como do N, foram divididas em cinco vezes na primeira época das águas e em sete vezes na segunda época das águas, sendo a primeira aplicação logo após o corte de uniformização de cada época e as outras após os demais cortes realizados.

Os DLS utilizados foram provenientes da granja de suínos da EV/UFG e passaram por um período de armazenamento de, no mínimo, 90 dias.
Os DLS foram transportados em tambores de $200 \mathrm{~L}$. Durante cada aplicação dos dejetos fazia-se a coleta de dois litros de amostra para análise do teor de nutrientes.

A forragem colhida em cada parcela foi acondicionada em sacos plásticos, identificada e pesada, sendo posteriormente retirada uma amostra representativa (500 g) e enviada ao Laboratório de Nutrição Animal Professor Francisco E. Galvão, do Departamento de Produção Animal (DPA), da Escola de Veterinária e Zootecnia (EVZ/UFG), onde foram secadas em estufa com ventilação de ar forçada, com temperaturas de 58 a $65^{\circ} \mathrm{C}$ por 48 horas, para determinação da matéria seca parcial, conforme metodologia de SILVA \& QUEIROZ (2002).

Após a secagem, as amostras foram moídas em moinho tipo Willey, com peneira de $1 \mathrm{~mm}$, armazenadas em saquinhos de plástico e identificadas. Em seguida, fez-se uma amostra composta do material resultante dos cortes realizados em função dos tratamentos utilizados em cada ano de avaliação. No Laboratório de Solos e Análises Foliares (EA/UFG), foram realizadas as análises químicas foliares para determinação dos teores de $\mathrm{N}$, $\mathrm{P}, \mathrm{K}, \mathrm{Ca}, \mathrm{Mg}, \mathrm{S}, \mathrm{Fe}, \mathrm{Cu}, \mathrm{Mn}$ e Zn. O N foi determinado pelo método de Kjeldahl, o $\mathrm{P}$, por colorimetria de azul de molibdênio, o K, por fotometria de chama de emissão, o $S$, por turbitimetria e o $\mathrm{Ca}$, o $\mathrm{Mg}$, o $\mathrm{Cu}$, o $\mathrm{Fe}$, o $\mathrm{Mn}$ e o $\mathrm{Zn}$, por espectofotometria de absorção atômica (MALAVOLTA et al., 1997).

O delineamento experimental utilizado foi 0 de blocos casualizados com parcelas subdivididas no tempo (GOMES, 1987). As parcelas foram constituídas pelos tratamentos de fertilização e as sub-parcelas pelos anos de avaliação. Fez-se uma amostra composta do material colhido nos cortes realizados em função dos tratamentos utilizados em cada ano de avaliação.

Os resultados obtidos no experimento foram tabulados em programas de planilhas eletrônicas e submetidos à análise de variância, Teste $\mathrm{F}$ e Teste de Tukey a 5\% de significância. Para a análise de dados utilizou-se o programa estatístico SAS (2001).

\section{RESULTADOS E DISCUSSÃO}

Observa-se pelos dados da Tabela 1 que não houve interação $(\mathrm{P}>0,05)$ entre os tratamentos de fertilização e os anos de avaliação, em relação aos teores médios de $\mathrm{N}$ foliares, no período das águas, permanecendo estes em torno de 1,5 a $1,6 \mathrm{dag} / \mathrm{kg}$. Tal resultado foi diferente do esperado, pois $\mathrm{N}$ foi adicionado ao solo em todos os tratamentos, com exceção do tratamento testemunha. No entanto, as 
concentrações de $\mathrm{N}$ foliares encontradas estão de acordo com as consideradas adequadas por Oliveira (2004) para Panicum maximum, que são de 1,5 a 2,5 $\mathrm{dag} / \mathrm{kg}$.

Constata-se também pelos dados da Tabela 1 que não houve interação $(\mathrm{P}>0,05)$ entre os tratamentos de fertilização e os anos de avaliação em relação aos teores médios de $\mathrm{P}$ foliares no período das águas. $\mathrm{O}$ tratamento mineral diferiu $(\mathrm{P}<0,05)$ dos demais, apresentando o menor teor médio de $\mathrm{P}$ foliar. Isso indica que devido ao fato de a forrageira ter produzido maior quantidade de MS nesse tratamento, possivelmente tenha propiciado maior efeito de diluição do $\mathrm{P}$ no tecido foliar. Para OLIVEIRA (2004), são adequados os teores de $P$ foliares, para Panicum maximum, variando de 0,1 a $0,3 \mathrm{dag} / \mathrm{kg}$, podendo-se considerar os teores de $\mathrm{P}$ encontrados nos diferentes tratamentos como adequados.

PINHEIRO (2006) observou aumento nos teores de $\mathrm{P}$ foliares do capim-Marandu de acordo com o aumento das doses de DLS aplicadas (100, 150 e $200 \mathrm{~m}^{3} / \mathrm{ha} / \mathrm{ano}$ ). ROSA et al. (2004a e 2004b) observaram que nem os tratamentos minerais nem os tratamentos orgânicos influenciaram os teores de $\mathrm{P}$ foliares encontrados no capim-Marandu, que permaneceram em torno de $0,3 \mathrm{dag} / \mathrm{kg}$.

Observa-se pelos dados da Tabela 2 que não houve interação $(\mathrm{P}>0,05)$ entre os tratamentos de fertilização e os anos de avaliação em relação aos teores médios de $\mathrm{K}$ foliares no período das águas. $\mathrm{O}$ tratamento testemunha e o tratamento mineral diferiram estatisticamente $(\mathrm{P}<0,05)$ entre si. Isso mostra que o tratamento mineral apresentou maior teor médio de $\mathrm{K}$ foliar devido ao fato de ter sido adicionado ao solo maior quantidade do nutriente neste tratamento. $\mathrm{O}$ segundo ano de avaliação apresentou teor médio de $\mathrm{K}$ foliar, estatisticamente $(\mathrm{P}<0,05)$ superior ao primeiro ano de avaliação. Explica-se tal fato pela lenta mineralização do K na forma orgânica nos tratamentos com DLS. De acordo com OLIVEIRA (2004), o K não está ligado a nenhum elemento químico, mas está presente em várias reações enzimáticas na planta. Segundo o mesmo autor, os teores foliares de $\mathrm{K}$ recomendados são de 1,5 a 3,0 dag/kg, para Panicum maximum. Portanto, sugere-se pelos dados da Tabela 3 que todos os tratamentos de adubação e o segundo ano de avaliação proporcionaram teores de $\mathrm{K}$ foliares adequados.

Verifica-se pelos dados da Tabela 3 que não houve interação $(\mathrm{P}>0,05)$ entre os tratamentos de fertilização e os anos de avaliação em relação aos teores médios de $\mathrm{Ca}$ e $\mathrm{Mg}$ foliares no período das águas. Ambos os minerais apresentaram teor médio foliar significativamente $(\mathrm{P}<0,05)$ superior no segundo ano de avaliação. Explica-se tal fato devido à lenta mineralização do Ca e Mg na forma orgânica nos tratamentos com DLS. Oliveira (2004) considera adequados teores de $\mathrm{Ca}$ e $\mathrm{Mg}$ foliares variando de 0,3 a $0,8 \mathrm{dag} / \mathrm{kg}$ e de 0,15 a $0,5 \mathrm{dag} / \mathrm{kg}$, respectivamente. Portanto, todos os tratamentos, bem como os anos de avaliação, apresentaram teores adequados desses nutrientes para Panicum maximum.

ROSA et al. (2004a e 2004b) e PINHEIRO (2006) não observaram influência da fertilização mineral ou orgânica $\left(100,150\right.$ e $\left.200 \mathrm{~m}^{3} / \mathrm{ha} / \mathrm{ano}\right)$ sobre os teores de $\mathrm{K}$, de $\mathrm{Ca}$ e de $\mathrm{Mg}$ foliares encontrados no capim-Marandu, que variaram de 1,0 a $4,0 \mathrm{dag} / \mathrm{kg}$, de 0,3 a $0,6 \mathrm{dag} / \mathrm{kg}$ e de 0,1 a 0,4 $\mathrm{dag} / \mathrm{kg}$, respectivamente.

Nota-se pelos dados da Tabela 3 que não houve interação $(\mathrm{P}>0,05)$ entre os tratamentos de fertilização e os anos de avaliação em relação aos teores médios de $\mathrm{S}$ foliares no período das águas. Explica-se tal fato pelo $\mathrm{S}$ ser parte constituinte dos aminoácidos, podendo ter comportamento semelhante aos teores de PB do capim-Mombaça, que não apresentaram diferença significativa entre os tratamentos, porém mantiveram-se elevados. Consideram-se adequados para Panicum maximum teores foliares de $S$ variando de 0,1 a $0,3 \mathrm{dag} / \mathrm{kg}$ (OLIVEIRA, 2004).

ROSA et al. (2004a) observaram que não houve nenhuma influência da fertilização mineral ou orgânica (100, 150 e $\left.200 \mathrm{~m}^{3} / \mathrm{ha} / \mathrm{ano}\right)$ sobre os teores de $\mathrm{S}$ foliares encontrados no capim-Marandu. ROSA et al. (2004b) observaram redução nos teores de S foliares encontrados no capim-Marandu com a utilização de DLS (100, 150 e $\left.200 \mathrm{~m}^{3} / \mathrm{ha} / \mathrm{ano}\right)$ de 0,21 para $0,14 \mathrm{dag} / \mathrm{kg}$.

Sabe-se que os minerais desempenham funções essenciais no organismo animal: participação como componentes estruturais dos tecidos corporais, manutenção do equilíbrio ácidobásico, pressão osmótica e permeabilidade das membranas celulares e ainda funcionam como ativadores de processos enzimáticos (TOKARNIA et al., 2006). Considerando a importância dos minerais, prevê-se que os teores médios de $\mathrm{P}, \mathrm{K}$, Ca e Mg foliares estariam suprindo de forma satisfatória as necessidades de bovinos de corte e de leite. Verificaram-se teores médios adequados de $\mathrm{S}$ para bovinos de corte, porém inadequados para bovinos de leite na fase de lactação. Porém, segundo TOKARNIA et al. (2006), não se conhece deficiência de S para bovinos mantidos em regime de pasto. 
TABELA 2 - Teores médios de nitrogênio (N), fósforo (P) e potássio (K) foliares do capim-Mombaça fertilizado com dejetos líquidos de suínos e adubo mineral no período das águas

\begin{tabular}{|c|c|c|c|c|c|c|c|c|c|}
\hline \multirow[b]{2}{*}{ Tratamentos } & \multicolumn{3}{|c|}{ N (dag/kg) } & \multicolumn{3}{|c|}{ P (dag/kg) } & \multicolumn{3}{|c|}{ K (dag/kg) } \\
\hline & Ano 1 & Ano 2 & Média & Ano 1 & Ano 2 & Média & Ano 1 & Ano 2 & Média \\
\hline Test (PK) & 1,64 & 1,63 & $1,64 \mathrm{a}$ & 0,47 & 0,44 & $0,45 \mathrm{a}$ & 1,27 & 1,53 & $1,40 \mathrm{~b}$ \\
\hline T M (NPK) & 1,60 & 1,67 & $1,64 \mathrm{a}$ & 0,29 & 0,39 & $0,34 \mathrm{~b}$ & 1,71 & 2,06 & $1,89 \mathrm{a}$ \\
\hline Т $100\left(\mathrm{~m}^{3} / \mathrm{ha}\right)$ & 1,55 & 1,56 & $1,55 \mathrm{a}$ & 0,50 & 0,45 & $0,47 \mathrm{a}$ & 1,32 & 1,91 & $1,61 \mathrm{ab}$ \\
\hline T $150\left(\mathrm{~m}^{3} / \mathrm{ha}\right)$ & 1,51 & 1,64 & $1,58 \mathrm{a}$ & 0,53 & 0,46 & $0,49 \mathrm{a}$ & 1,13 & 2,13 & $1,63 \mathrm{ab}$ \\
\hline Т $200\left(\mathrm{~m}^{3} / \mathrm{ha}\right)$ & 1,62 & 1,66 & $1,64 \mathrm{a}$ & 0,52 & 0,47 & $0,49 \mathrm{a}$ & 1,11 & 1,84 & $1,47 \mathrm{ab}$ \\
\hline Média & 1,63 A & $1,58 \mathrm{~A}$ & & $0,46 \mathrm{~A}$ & $0,44 \mathrm{~A}$ & & 1,31 B & $1,89 \mathrm{~A}$ & \\
\hline CV (\%) & & 8,08 & & & 13,09 & & & 28,14 & \\
\hline
\end{tabular}

$\mathrm{a}>\mathrm{b}(\mathrm{p}<0,05) ; \mathrm{A}>\mathrm{B}(\mathrm{p}<0,05) ;$ As letras minúsculas comparam as médias dentro da coluna e as maiúsculas, as médias dentro de linha; $\mathrm{dag} / \mathrm{kg}=\%$

TABELA 3 - Teores médios de cálcio (Ca), magnésio (Mg) e enxofre (S) foliares do capim-Mombaça fertilizado com dejetos líquidos de suínos e adubo mineral no período das águas

\begin{tabular}{|c|c|c|c|c|c|c|c|c|c|}
\hline \multirow[b]{2}{*}{ Tratamentos } & \multicolumn{3}{|c|}{ Ca (dag/kg) } & \multicolumn{3}{|c|}{ Mg (dag/kg) } & \multicolumn{3}{|c|}{ S (dag/kg) } \\
\hline & Ano 1 & Ano 2 & Média & Ano 1 & Ano 2 & Média & Ano 1 & Ano 2 & Média \\
\hline Test (PK) & 0,59 & 0,72 & $0,65 \mathrm{a}$ & 0,30 & 0,28 & $0,29 \mathrm{a}$ & 0,18 & 0,18 & $0,18 \mathrm{a}$ \\
\hline T M (NPK) & 0,55 & 0,80 & $0,67 \mathrm{a}$ & 0,23 & 0,32 & $0,27 \mathrm{a}$ & 0,20 & 0,17 & $0,18 \mathrm{a}$ \\
\hline T $100\left(\mathrm{~m}^{3}\right)$ & 0,66 & 0,74 & $0,70 \mathrm{a}$ & 0,31 & 0,36 & $0,33 \mathrm{a}$ & 0,18 & 0,17 & $0,18 \mathrm{a}$ \\
\hline T $150\left(\mathrm{~m}^{3}\right)$ & 0,70 & 0,83 & $0,77 \mathrm{a}$ & 0,30 & 0,34 & $0,32 \mathrm{a}$ & 0,18 & 0,18 & $0,18 \mathrm{a}$ \\
\hline T $200\left(\mathrm{~m}^{3}\right)$ & 0,62 & 0,82 & $0,72 \mathrm{a}$ & 0,21 & 0,32 & $0,26 \mathrm{a}$ & 0,18 & 0,17 & $0,18 \mathrm{a}$ \\
\hline Média & $0,62 \mathrm{~B}$ & $0,78 \mathrm{~A}$ & & $0,27 \mathrm{~B}$ & $0,32 \mathrm{~A}$ & & $0,18 \mathrm{~A}$ & $0,17 \mathrm{~A}$ & \\
\hline CV (\%) & & 16,44 & & & 24,77 & & & 14,31 & \\
\hline
\end{tabular}

A>B (P<0,05); As letras minúsculas comparam as médias dentro da coluna e as maiúsculas, as médias dentro de linha; dag/kg = \%

Percebe-se pelos dados das Tabelas 4 e 5 que não houve interação $(\mathrm{P}>0,05)$ entre os tratamentos de fertilização e os anos de avaliação em relação aos teores médios de $\mathrm{Cu}, \mathrm{Fe}$, Mn e Zn foliares no período das águas. $\mathrm{O} \mathrm{Cu}$, o $\mathrm{Fe}$ e o $\mathrm{Zn}$ apresentaram teores médios foliares estatisticamente $(\mathrm{P}<0,05)$ superiores no segundo ano de avaliação. Considerando o $\mathrm{Cu}$, ENSMINGER et al. (1990) afirmaram que a disponibilidade do nutriente na planta é dependente de seu conteúdo no solo; entretanto, a concentração de $\mathrm{Cu}$ presente no tratamento mineral e no tratamento testemunha, que não receberam o nutriente, não apresentou diferença significativa
$(\mathrm{P}>0,05)$ dos tratamentos com utilização de DLS. Tal fato pode significar que a planta não conseguiu absorver o $\mathrm{Cu}$ excedente que foi aplicado via DLS, permitindo-se afirmar que a maior parte do nutriente ainda esteja complexada à matéria orgânica, podendo, assim, haver acúmulo do nutriente no solo, o que explica o fato de, no segundo ano de avaliação, ter apresentado teor médio mais elevado. Os teores de $\mathrm{Cu}$ recomendados por OLIVEIRA (2004) para Panicum maximum são de 4 a $14 \mathrm{mg} / \mathrm{kg}$. Assim, conclui-se que todos os tratamentos, bem como os dois anos de avaliação, apresentaram teores de $\mathrm{Cu}$ foliares adequados.

TABELA 4 - Teores médios de cobre ( $\mathrm{Cu}$ ) e de ferro (Fe) foliares do capim- Mombaça fertilizado com dejetos líquidos de suínos e adubo mineral no período das águas

\begin{tabular}{ccccccc}
\hline & \multicolumn{3}{c}{ Cu $(\mathrm{mg} / \mathrm{kg})$} & \multicolumn{3}{c}{ Fe $(\mathrm{mg} / \mathrm{kg})$} \\
\cline { 2 - 7 } Tratamentos & Ano 1 & Ano 2 & Média & Ano 1 & Ano 2 & Média \\
\hline Test (PK) & 5,18 & 13,72 & $9,45 \mathrm{a}$ & 204,62 & 385,97 & $295,30 \mathrm{a}$ \\
T M (NPK) & 5,02 & 10,29 & $12,11 \mathrm{a}$ & 218,86 & 377,12 & $297,99 \mathrm{a}$ \\
T $100\left(\mathrm{~m}^{3}\right)$ & 5,31 & 15,05 & $10,18 \mathrm{a}$ & 226,62 & 334,62 & $280,63 \mathrm{a}$ \\
T $150\left(\mathrm{~m}^{3}\right)$ & 5,57 & 14,25 & $9,91 \mathrm{a}$ & 203,97 & 371,81 & $287,89 \mathrm{a}$ \\
T $200\left(\mathrm{~m}^{3}\right)$ & 5,72 & 15,36 & $10,54 \mathrm{a}$ & 220,73 & 302,35 & $261,54 \mathrm{a}$ \\
Média & $5,36 \mathrm{~B}$ & $15,51 \mathrm{~A}$ & & $214,97 \mathrm{~B}$ & $354,38 \mathrm{~A}$ & \\
CV $(\%)$ & & 34,80 & & & 28,10 & \\
\hline
\end{tabular}

$\mathrm{A}>\mathrm{B}(\mathrm{P}<0,05) ;$ As letras minúsculas comparam as médias dentro da coluna e as maiúsculas, as médias dentro de linha; $\mathrm{mg} / \mathrm{kg}=$ ppm 
Considerando os teores foliares de Fe encontrados, observa-se que não houve influência na concentração do nutriente no solo e na planta, pois na aplicação de DLS foram colocadas no solo altas concentrações de Fe; no entanto, nenhum dos tratamentos de fertilização diferiu estatisticamente $(\mathrm{P}<0,05)$ entre si. De acordo com os dados apresentados na Tabela 5, os teores médios de Fe foliares encontrados estão acima do limite recomendado por OLIVEIRA (2004) para Panicum maximum, que são de 50 a 200 mg/kg. Em relação ao $\mathrm{Mn}$, observa-se que os valores encontrados para o tratamento testemunha e para os tratamentos orgânicos foram significativamente
$(\mathrm{P}<0,05)$ menores que os encontrados para o tratamento mineral, com exceção da aplicação de $200 \mathrm{~m}^{3} / \mathrm{ha} / \mathrm{ano}$ de DLS. Explica-se tal fato pela interação entre os nutrientes $\mathrm{Fe}$ e $\mathrm{Mn}$, pois altas concentrações de Fe podem induzir a uma menor absorção de Mn (Resende, 2005). Esse fato explica o baixo teor médio de $\mathrm{Mn}$ foliar encontrado no segundo ano de avaliação. Para OLIVEIRA (2004), os teores de $\mathrm{Mn}$ foliares recomendados para Panicum maximum variam de 40 a $200 \mathrm{mg} / \mathrm{kg}$. Sendo assim, considerando todos os tratamentos, bem como os anos de avaliação, os teores foram adequados.

TABELA 5 - Teores médios de manganês (Mn) e de zinco (Zn) foliares do capim- Mombaça fertilizado com dejetos líquidos de suínos e adubo mineral no período das águas

\begin{tabular}{ccccccc}
\hline & \multicolumn{3}{c}{ Mn $(\mathrm{mg} / \mathrm{kg})$} & & Zn $(\mathrm{mg} / \mathrm{kg})$ \\
\hline Tratamentos & Ano 1 & Ano 2 & Média & Ano 1 & Ano 2 & Média \\
\hline Test (PK) & 101,21 & 54,81 & $78,01 \mathrm{~b}$ & 15,88 & 21,98 & $18,93 \mathrm{a}$ \\
T M $(\mathrm{NPK})$ & 123,80 & 83,40 & $103,60 \mathrm{a}$ & 17,36 & 24,08 & $20,72 \mathrm{a}$ \\
T $100\left(\mathrm{~m}^{3}\right)$ & 103,37 & 45,98 & $74,68 \mathrm{~b}$ & 17,17 & 28,60 & $22,88 \mathrm{a}$ \\
T $150\left(\mathrm{~m}^{3}\right)$ & 102,95 & 51,67 & $77,13 \mathrm{~b}$ & 17,53 & 27,06 & $22,30 \mathrm{a}$ \\
T $200\left(\mathrm{~m}^{3}\right)$ & 104,40 & 58,16 & $81,28 \mathrm{ab}$ & 19,48 & 24,45 & $21,96 \mathrm{a}$ \\
\hline Média & $107,14 \mathrm{~A}$ & $58,80 \mathrm{~B}$ & & $17,49 \mathrm{~B}$ & $25,23 \mathrm{~A}$ & \\
\hline CV $(\%)$ & 25,48 & & 25,54 &
\end{tabular}

a $>\mathrm{b}(\mathrm{P}<0,05) ; \mathrm{A}>\mathrm{B}(\mathrm{P}<0,05) ;$ As letras minúsculas comparam as médias dentro da coluna e as maiúsculas, as médias dentro de linha; $\mathrm{mg} / \mathrm{kg}=\mathrm{ppm}$

Considerando os teores de $\mathrm{Zn}$ foliares, o segundo ano de avaliação apresentou teor médio de $\mathrm{Zn}$ foliar estatisticamente superior $(\mathrm{P}<0,05)$ ao primeiro ano de avaliação. Sabe-se que o pH alcalino prejudica a disponibilidade e absorção do Zn; no entanto, este fato não ocorreu no experimento. Isto explica o segundo ano ter apresentado teor superior, pois o $\mathrm{pH}$ do solo estava ácido, o que provavelmente contribuiu para maior disponibilidade de $\mathrm{Zn}$, aumentando a sua absorção pela planta. Para OLIVEIRA (2004) os teores adequados de Zn para Panicum maximum variam de 20 a $50 \mathrm{mg} / \mathrm{kg}$. Sendo assim, o segundo ano de avaliação, bem como os tratamentos minerais e orgânicos, produziram teores de $\mathrm{Zn}$ adequados.

PINHEIRO (2006) avaliou o efeito da utilização de DLS (100, 150 e $\left.200 \mathrm{~m}^{3} / \mathrm{ha} / \mathrm{ano}\right)$ na recuperação de pastagens de Brachiaria brizantha cv. Marandu e constatou que houve um incremento na concentração de $\mathrm{Cu}$ e de $\mathrm{Zn}$, com o aumento das doses de DLS, sendo que os nutrientes variaram de 8 a $9 \mathrm{mg} / \mathrm{kg}$ e de 25 a $28 \mathrm{mg} / \mathrm{kg}$, respectivamente. Observou também que não houve efeito significativo entre os tratamentos adotados para os teores de $\mathrm{Fe}$ foliares, permanecendo em torno de 174 a 187 $\mathrm{mg} / \mathrm{kg}$. Em relação ao $\mathrm{Mn}$, constatou que os tratamentos orgânicos produziram teores significativamente inferiores quando comparados com os minerais, variando de 66 a $105 \mathrm{mg} / \mathrm{kg}$.

No entanto, ROSA et al. (2004 a, e 2004 b) observaram que os teores de $\mathrm{Cu}$ (6 a $8 \mathrm{mg} / \mathrm{kg}$ ), Fe (182 a 238 mg/kg) e Zn (18 a 41 mg/kg) não foram influenciados nem pela fertilização mineral, nem pela fertilização orgânica. Os tratamentos minerais (PK e NPK) possibilitaram maiores teores de Mn foliar (variando de 102 a $145 \mathrm{mg} / \mathrm{kg}$ ).

Concluiu-se que os teores médios de $\mathrm{Cu}$, de $\mathrm{Fe}$ e de Mn suprem de forma satisfatória as necessidades de bovinos de corte e de bovinos de leite. Porém os teores de Fe encontrados (Tabela 5) estão bem acima do limite, que é em torno de 50 $\mathrm{mg} / \mathrm{kg}$. Com relação ao nível de toxidez, segundo o 
NRC (1996), este valor é de $1000 \mathrm{mg} / \mathrm{kg}$; portanto, todos os valores encontrados estão abaixo de tal nível. Os teores médios de $\mathrm{Zn}$ foliares encontrados estão abaixo do recomendado para bovinos de corte e para bovinos de leite. A deficiência de Zn pode causar em bovinos um distúrbio (paraqueratose hereditária) que prejudica a absorção de Zn pela mucosa intestinal (TOKARNIA et al., 2006). No entanto, o problema pode ser resolvido utilizando-se a suplementação mineral.

\section{CONCLUSÃO}

A dose de $200 \mathrm{~m}^{3} / \mathrm{ha} /$ ano pode substituir a fertilização mineral do tratamento NPK.

\section{REFERÊNCIAS}

ADELI, A..; VARCO, J. J. Swine lagoon as a source of nitrogen and phosphorus for summer forage grasses. Agronomy Journal. v. 93, n. 5, p. 1174-1181,2001.

ECHBERG, B. Effluent engineers. Pig International. v.33, n.9, Mount Morris, p. 13-15, October. 2003.

ENSMINGER, M.E., OLDFIELD, J.E., HEINEMANN, W.W. 1990. Feeds and nutrition (Formely, Feeds and Nutritiom - complete) 2. ed. The Ensminger Publishing Company. 1544p.

FAVORETTO, V. Metodologia de avaliação de forrageiras. Jaboticabal: FCAV, 1994.

GOMES, F. P. Curso de Estatística Experimental. 12 ed. Editora Distribuidora. Escola Superior de Agricultura Luiz de Queiroz - Universidade de São Paulo, Piracicaba, 1987. 467p.

JANK, L.; SAVIDAN, Y. SOUZA, M.T. de; COSTA, J.C.G. Avaliação do germoplasma de Panicum maximum introduzido da África. 1. Produção forrageira. Revista Brasileira de Zootecnia, Viçosa, v.23, n.3, p.433-440, 1994.

KONZEN, E. A. Viabilidade ambiental e soluções de mercado para utilização dos dejetos em uma granja de suínos. In: RODADA GOIANA DE TECNOLOGIA EM MANEJO DE SUÍNOS, 10, Goiânia, 2006. Anais...Goiânia: AGS, 2006. Disponível em: www.cnpms.embrapa.br/publicacoes/publica/2006/.../Doc _59.pdf

MALAVOLTA, E., LIEM, T.H., OLIVEIRA, S.A. Avaliação do estado nutricional das plantas. Princípios e aplicações. 2. ed. Piracicaba: Potafos, 1997. 319p.
MARRIEL, I. V.; KONZEN, E. A.; ALVARENGA, R. C.; SANTOS, H. L. Tratamento e utilização de resíduos orgânicos. Informe Agropecuário, Belo Horizonte, v. 13, n. 147, p.24-36, 1987.

MENEZES, J. F. S.; ALVARENGA, R.C.; ANDRADE, C. L. T.; KONZEN, E. A.; PIMENTA, F. F. Aproveitamento de resíduos orgânicos para a produção de grãos em sistema de plantio direto e avaliação do impacto ambiental. Revista Plantio Direto. n. 73, Ano XII, p. 3035, 2003.

NATIONAL RESEARCH COUNCIL - NRC. Nutrient requirements of beef cattle. 7.ed. Washington, D.C.: National Academy Press, 1996. 242p.

OLIVEIRA, S. A. Análise foliar. In: SOUZA, D. M. G.; LOBATO, E. Cerrado: correção do solo e adubação. 2. ed. EMBRAPA Informação Tecnológica, Brasília:DF, 2004, cap. 10, p.245-256.

PINHEIRO, E.P. Produção de massa seca e composição químico-bramatológica da Brachiaria brizantha stapf cv. maramdu fertilizada com dejetos líquidos de suínos e adubos mineral 2006. 39 f. Dissertação (Mestrado em Medicina Veterinária) - Escola de Veterinária, Universidade Federal de Goiás, Goiânia. Disponível em: www.bc.ufg.br/sophia/index.php?codigo_sophia=99360

RESENDE, A. V. de. Micronutrientes na agricultura brasileira: disponibilidade, utilização e perspectivas. Série Estudos e Documentos. Coletânea Fertilizantes, 9. Centro de Tecnologia Mineral - Ministério da Ciência e Tecnologia, 2005. 37p.

ROSA, B.; BARNABÉ, F. H. G. A.; SILVA, L. T.; NAVES, M. A. T.; RAMOS, C. S.; FREITAS, K. R.; MOZENA, W. L. Produção e composição químicobromatológica do capim Braquiarão cv. Marandu fertilizado com diferentes doses de dejetos líquidos de suínos. In: REUNIÃO ANUAL DA SOCIEDADE BRASILEIRA DE ZOOTECNIA, 41., 2004 a. Campo Grande. Anais... Campo Grande, CD ROM. Disponível em

http://www.sbz.org.br/reuniaoanual/anais/arq_reuniao_an ual/sbz2004.rar. Acesso em janeiro 2008

ROSA, B.; NAVES, M. A. T.; RAMOS, C. S.; FREITAS, K. R.; HEINEMANN, A. B.; MOZENA, W. L. Utilização de dejetos líquidos de suínos na produção e composição químico-bromatológica do capim Braquiarão (Brachiaria brizantha cv. Marandu) In: REUNIÃO ANUAL DA SOCIEDADE BRASILEIRA DE ZOOTECNIA, 41., 2004 b. Campo Grande. Anais...Campo Grande, CD ROM. Disponível em http://www.sbz.org.br/reuniaoanual/anais/arq_reuniao_an ual/sbz2004.rar. Acesso em janeiro 2008 
SAS INSTITUTE. Statistical Analisys Sistem. The SAS sistem for windows. Cary SAS Institute, 2001.

SILVA, D.J., QUEIROZ, A. C. Análise de Alimentos (métodos químicos e biológicos). 3.ed., Viçosa: Imprensa Universitária da UFV, 2002, 235 p.

TOKARNIA, C. H.; DOBEREINER, J.; PEIXOTO, P. V. Deficiências minerais em animais de fazenda, principalmente bovinos. Sanidade Animal. Brasília, DF, p.41-52. 2006. In: DOBEREINER, J. EMBRAPA Informações Tecnológicas. Colégio Brasileiro de Patologia Animal. EMBRAPA Informações Tecnológicas, 2005. 282 p.

VILELA, L.; SOARES, W. V.; SOUSA, D. M. G.; MACEDO, M. C. M. Calagem e adubação para pastagem. In: SOUZA, D. M. G.; LOBATO, E. Cerrado: correção do solo e adubação. 2. ed.EMBRAPA Informação Tecnológica, Brasília:DF, 2004, cap. 14, p.367-384.

Protocolado em: 07 fev. 2008. Aceito em: 10 maio 2011 